\title{
Localization of Gravity and Bulk Matters on a Thick Anti-de Sitter Brane
}

\author{
Yu-Xiao Liu*, Heng Guo ${ }^{\dagger}$, Chun-E Fu ${ }^{\ddagger}$, Hai-Tao Li ${ }^{\S}$ \\ Institute of Theoretical Physics,Lanzhou University, \\ Lanzhou 730000, People's Republic of China
}

\begin{abstract}
In this paper, we investigate the localization and the mass spectra of gravity and various bulk matter fields on a thick anti-de Sitter (AdS) brane, by presenting the mass-independent potentials of the Kaluza-Klein (KK) modes in the corresponding Schrödinger equations. For gravity, the potential of the KK modes tends to infinity at the boundaries of the extra dimension, which leads to an infinite number of the bound KK modes. Although the gravity zero mode cannot be localized on the AdS brane, the massive modes are trapped on the brane. The scalar perturbations of the thick AdS brane have been analyzed, and the brane is stable under the scalar perturbations. For spin-0 scalar fields and spin-1 vector fields, the potentials of the KK modes also tend to infinity at the boundaries of the extra dimension, and the characteristic of the localization is the same as the case of gravity. For spin-1/2 fermions, by introducing the usual Yukawa coupling $\eta \bar{\Psi} \phi \Psi$ with the positive coupling constant $\eta$, the four-dimensional massless left-chiral fermion and massive Dirac fermions are obtained on the AdS thick brane.
\end{abstract}

PACS numbers: 04.50.-h, 11.27.+d

\footnotetext{
*liuyx@lzu.edu.cn

$\dagger$ guoh2009@lzu.cn,Corresponding author

$\ddagger$ fuche08@lzu.cn

$\S$ liht07@lzu.cn
} 


\section{INTRODUCTION}

The idea that our observed four-dimensional Universe might be a 3-brane, embedded in a higher dimensional space-time (the bulk), provides new insights into solving the gauge hierarchy and cosmological constant problems [1 8 ]. In the Randall-Sundrum (RS) brane world model, the zero mode of gravity is localized on the brane, which reproduces the standard Newtonian gravity on the brane [5]. But in this model, the brane is very ideal because its thickness is neglected. In the most fundamental theory, there seems to exist a minimum scale of length, thus the thickness of a brane should be considered in more realistic field models. For this reason, more natural thick brane scenarios have been investigated [9 41]. For some comprehensive reviews about thick branes, see Refs. [42-47].

In brane world theory, only gravity is free to propagate in both the brane and bulk space-time, however, all the matter fields (electromagnetic, Yang-Mills, etc.) in our fourdimensional Universe are confined to the 3-brane with no contradiction with present gravitational experiments. Hence, in order to build up the standard model, various bulk matter fields should be localized on branes by a natural mechanism. Generally, the massless scalar fields [48] could be trapped on branes of different types. Spin-1 Abelian vector fields can be localized on the RS brane in some higher dimensional cases [49] or on the thick de Sitter brane and Weyl thick brane [50]. It is important to study the localization problem of the spin- $1 / 2$ fermions. Without introducing the scalar-fermion coupling, fermions cannot be localized on branes in five and six dimensions [48 71]. In some cases, there may exist a single bound state and a continuous gapless spectrum of massive fermion Kaluza-Klein (KK) modes [25, 50]. In some other cases, one can obtain finite discrete KK modes (mass gap) and a continuous gapless spectrum starting at a positive $m^{2}[28,63,64]$.

Most investigations mentioned above mainly focused on flat branes. In Ref. [18], the solutions of de Sitter (dS) and anti-de Sitter (AdS) 3-branes were presented, and the localization of gravity on the dS 3-brane was also studied. In this paper, in order to show the rich structures of the AdS 3-brane from the other points of view, we would like to investigate the localization problem of gravity and various spin matter fields (scalars, vectors and fermions) on the brane. For this AdS 3-brane solution, the behavior of the warp factor is related with a parameter $\delta$. For $\delta>1$, the warp factor is divergent at $z= \pm z_{b}$ the boundaries of the extra dimension, and the energy density has a lump at $z \approx 0$; i.e., the brane is located at 
$z \approx 0$. For $\delta<0$, the warp factor tends to zero far away from the brane, but the energy density has no lump at $z \approx 0$ and diverges at $z= \pm z_{b}$, so this configuration cannot be considered as a thick brane. Hence, in this paper, we only consider that the parameter is constrained as $\delta>1$. For gravity, the zero mode is not localized on the thick AdS brane; however, the massive KK modes can be localized on the brane, and the mass spectrum consists of an infinite number of discrete bound states. For free scalar fields and vector fields, all the KK modes are bound states and the massive modes, which could be trapped on the brane. For spin- $1 / 2$ fermions coupling with the background scalar by the usual Yukawa coupling $\eta \bar{\Psi} \phi \Psi$, the left-chiral fermion massless mode can be localized on the brane, and both left- and right-chiral fermions are also localized on the brane, which can constitute the four-dimensional massive Dirac fermions.

The organization of this paper is as follows: In Sec. III, we first review the thick AdS 3-brane in five-dimensional space-time. Then, in Sec. III, we investigate the localization and the mass spectra of gravity on the thick AdS 3-brane. In Sec. IV, the localization and the mass spectra of various bulk matter fields are investigated. For scalars and vectors, we give the analytical expressions for the KK modes and the mass spectrums. For fermions, the analytical formulations of the massless modes are also obtained. Finally, our conclusion is given in Sec. $\nabla$.

\section{REVIEW OF THE THICK ANTI-DE SITTER 3-BRANE}

We start with the following five-dimensional action of thick branes, which are generated by a real scalar field $\phi$ with a scalar potential $V(\phi)$,

$$
S=\int d^{5} x \sqrt{-g}\left[\frac{1}{2 \kappa_{5}^{2}} R-\frac{1}{2} g^{M N} \partial_{M} \phi \partial_{N} \phi-V(\phi)\right],
$$

where $R$ is the five-dimensional scalar curvature and $\kappa_{5}^{2}=8 \pi G_{5}$ with $G_{5}$ the five-dimensional Newton constant. The most general five-dimensional metric compatible with an $\mathrm{AdS}_{4}$ symmetry can be taken as

$$
\begin{aligned}
d s^{2} & =g_{M N} d x^{M} d x^{N}=\mathrm{e}^{2 A(z)}\left[\hat{g}_{\mu \nu}(x) d x^{\mu} d x^{\nu}+d z^{2}\right] \\
& =\mathrm{e}^{2 A(z)}\left[\mathrm{e}^{2 H x_{3}}\left(-d t^{2}+d x_{1}^{2}+d x_{2}^{2}\right)+d x_{3}^{2}+d z^{2}\right],
\end{aligned}
$$

where $\mathrm{e}^{2 A(z)}$ is the warp factor, $H$ is the $\mathrm{AdS}_{4}$ parameter and $z$ stands for the extra coordinate. We suppose that the scalar field is a function of $z$ only, i.e., $\phi=\phi(z)$. The 
four-dimensional cosmology constant can be expressed as $\Lambda_{4}=-3 H^{2}$. By considering the action (11) and the metric (2), the filed equations reduce to the following coupled nonlinear differential equations:

$$
\begin{aligned}
\phi^{\prime 2} & =\frac{3}{\kappa_{5}^{2}}\left(A^{\prime 2}-A^{\prime \prime}+H^{2}\right), \\
V(\phi) & =-\frac{3 \mathrm{e}^{-2 A}}{2 \kappa_{5}^{2}}\left(3 H^{2}+3 A^{\prime 2}+A^{\prime \prime}\right), \\
\frac{d V(\phi)}{d \phi} & =\mathrm{e}^{-2 A}\left(3 A^{\prime} \phi^{\prime}+\phi^{\prime \prime}\right),
\end{aligned}
$$

where the prime denotes the derivative with respect to $z$. These equations are not independent. The relationship between these equations was discussed in [14].

Here we set $\kappa_{5}^{2}=1$. A thick AdS brane solution in five-dimensional space-time for the potential

$$
V(\phi)=-\frac{3(1+3 \delta) H^{2}}{2 \delta} \cosh ^{2(1-\delta)}\left(\frac{\phi}{\phi_{0}}\right)
$$

was found in Ref. [18]:

$$
\begin{aligned}
& A(z)=-\delta \ln |\cos \bar{z}|, \\
& \phi(z)=\phi_{0} \operatorname{arcsinh}(\tan \bar{z}),
\end{aligned}
$$

where

$$
\begin{aligned}
\phi_{0} & \equiv \sqrt{3 \delta(\delta-1)}, \\
\bar{z} & \equiv \frac{H}{\delta} z,
\end{aligned}
$$

and the parameter $\delta$ satisfies $\delta>1$ or $\delta<0$. The range of the extra dimension is $-z_{b} \leq$ $z \leq z_{b}$ with $z_{b}=\left|\frac{\delta \pi}{2 H}\right|$. The metric with this choice of $A(z)$ has a naked singularity at $\pm z_{b}$. This singularity is very similar to the one in Ref. [11] and the one encountered in the AdS flow to $N=1$ super Yang-Mills theory [72]. Gremm supported that this might indicate that it can be resolved either by lifting the five-dimensional geometry to ten dimensions or by string theory [11]. From (6), it can be seen that the background scalar field diverges at the boundaries of the extra dimension $\pm z_{b}$. In Ref. [11], this divergence can indicate that the compactification manifold shrinks to zero or becomes infinitely large, so that the five-dimensional truncation comes to be invalid. There are some examples that singularities in five dimensions actually correspond to nonsingular ten-dimensional geometries [73]. 
As is well-known, the energy-momentum tensor for a scalar field is energetically equivalent to an anisotropic fluid, $T_{M N}=-\rho\left(g_{M N}+z_{M} z_{N}\right)+p z_{M} z_{N}$, where $z_{M}=\mathrm{e}^{A} \delta_{M}^{5}$ and

$$
\begin{aligned}
\rho & \equiv \frac{1}{2}\left[\mathrm{e}^{-2 A} \phi^{2}+2 V(\phi)\right] \\
& =-\frac{3 H^{2}(1+\delta)}{\delta} \cos ^{2(\delta-1)}(\bar{z}), \\
p & \equiv \frac{1}{2}\left[\mathrm{e}^{-2 A} \phi^{2}-2 V(\phi)\right] \\
& =6 H^{2} \cos ^{2(\delta-1)}(\bar{z}) .
\end{aligned}
$$

From the above expressions, it can be shown that, for $\delta<0$ or $\delta>1$, the corresponding energy-momentum tensor satisfies the weaker energy condition $T_{M N} \zeta^{M} \zeta^{N} \geq 0$ with $\zeta^{M}$ an arbitrary null vector. For $-1<\delta<0$, the weak energy condition $T_{M N} \xi^{M} \xi^{N} \geq 0$ is satisfied, where $\xi^{M}$ is an arbitrary future-directed timelike or null vector, and for $-\frac{1}{3}<\delta<0$ the dominant energy condition $T_{M N} \xi^{M} \eta^{N} \geq 0$ with $\eta^{M}$ an arbitrary future-directed timelike or null vector is satisfied. It is clear that, for $\delta>1$, the energy density $\rho$ has a lump at $|z| \approx 0$ and tends to zero at the boundaries of the extra dimension $z= \pm z_{b}$, which shows that the thick 3-brane locates at $|z| \approx 0$. However, for $\delta<0$, the energy density has no lump at $z \approx 0$ and diverges at $\pm z_{b}$, and it is difficult to regard this configuration as a thick brane, so in the following sections, we only consider the case of $\delta>1$.

\section{LOCALIZATION OF GRAVITY ON THE THICK ANTI-DE SITTER BRANE}

In this section, the localization of gravity will be investigated by presenting the massindependent potential of the KK modes for gravitons in the corresponding Schrödinger equation.

Let us consider the following perturbed metric:

$$
d s^{2}=\left(g_{M N}+\delta g_{M N}\right) d x^{M} d x^{N} .
$$

As Ref. [5], we impose the axial gauge constraint $\delta g_{5 M}=0$, and then write the total metric in the form

$$
d s^{2}=\mathrm{e}^{2 A(z)}\left[\left(\hat{g}_{\mu \nu}+h_{\mu \nu}\right) d x^{\mu} d x^{\nu}+d z^{2}\right],
$$

where $h_{\mu \nu}$ denotes the metric perturbation. Then, under the transverse-traceless gauge condition $h^{\mu}{ }_{\mu}=\partial^{\mu} h_{\mu \nu}=0$, the equation for the perturbation $h_{\mu \nu}$ takes the following form [13]

$$
\left[\partial_{z}^{2}+3 A^{\prime}(z) \partial_{z}+\square+2 H^{2}\right] h_{\mu \nu}\left(x^{\alpha}, z\right)=0,
$$


where $\square \equiv \hat{g}^{\mu \nu} \nabla_{\mu} \nabla_{\nu}$ and $\nabla_{\mu}$ denotes the covariant derivative with respect to the fourdimensional metric $\hat{g}_{\mu \nu}$. By making use of the KK decomposition

$$
h_{\mu \nu}\left(x^{\alpha}, z\right)=\mathrm{e}^{-\frac{3}{2} A(z)} \epsilon_{\mu \nu}\left(x^{\alpha}\right) \varphi(z)
$$

with $\epsilon_{\mu \nu}\left(x^{\alpha}\right)$ satisfying the transverse-traceless condition, from Eq. (13) we can get the following four-dimensional equation

$$
\square \epsilon_{\mu \nu}\left(x^{\alpha}\right)+2 H^{2} \epsilon_{\mu \nu}\left(x^{\alpha}\right)=m^{2} \epsilon_{\mu \nu}\left(x^{\alpha}\right),
$$

where $m$ is the mass of the KK modes. The Schrödinger-like equation for the fifthdimensional sector is also obtained

$$
\left(-\partial_{z}^{2}+V_{\mathrm{QM}}\right) \varphi(z)=m^{2} \varphi(z)
$$

with the potential

$$
\begin{aligned}
V_{\mathrm{QM}} & =\frac{3}{2} A^{\prime \prime}(z)+\frac{9}{4} A^{\prime 2}(z) \\
& =\frac{3 H^{2}}{4 \delta}\left[(2+3 \delta) \sec ^{2} \bar{z}-3 \delta\right]
\end{aligned}
$$

The shapes of the potential for the KK modes are plotted in Fig. 1, The potential $V_{\mathrm{QM}}(z)$ has minimum $\frac{3 H^{2}}{2 \delta}$ (positive value) at the location of the brane $(z=0)$, and tends to positive infinity at the boundaries of the fifth dimension $\left(z= \pm z_{b}\right)$, so the potential has no negative value at the location of the brane, and it cannot trap the zero mode of gravity, but it can trap the massive modes. Eq. (16) can be turned into the following equation with $E_{n}^{2}=m_{n}^{2}+\frac{9 H^{2}}{4}$ :

$$
\left[-\partial_{z}^{2}+\frac{3 H^{2}}{4 \delta}(2+3 \delta) \sec ^{2} \bar{z}\right] \varphi_{n}(z)=E_{n}^{2} \varphi_{n}(z) .
$$

When the parameter $\delta$ satisfies $\delta>1$, the solutions of the gravity KK modes are found to be

$$
\begin{aligned}
\varphi_{n}(z) \propto \quad & { }_{2} \mathrm{~F}_{1}\left[1-n, 1+n+3 \delta, \frac{3(1+\delta)}{2}, \frac{1-\sin \bar{z}}{2}\right] \\
& \times \cos ^{1+\frac{3 \delta}{2}}(\bar{z})
\end{aligned}
$$

where $n=1,2, \cdots$. Then the mass spectrum of bound states is found to be

$$
E_{n}=\frac{H}{\delta}\left(n+\frac{3}{2} \delta\right)
$$


or

$$
m_{n}=\frac{H}{\delta} \sqrt{n(n+3 \delta)}
$$

It can be found that the ground state is the massive mode with $m_{1}=\frac{H}{\delta} \sqrt{1+3 \delta}$. All the KK modes are bound states, and localized on the thick AdS brane. The mass spectrum of the KK modes is discrete. The shapes of the gravity KK modes with lower mass are shown in Fig. 2,

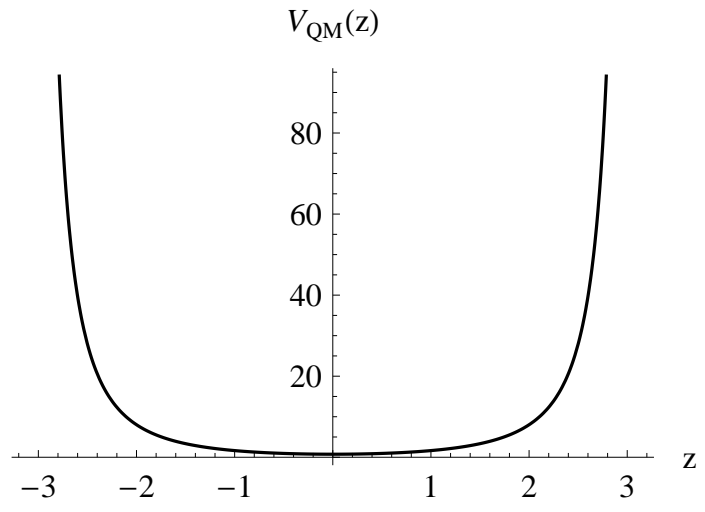

(a)

FIG. 1: The shape of the potential of the gravity KK modes $V_{\mathrm{QM}}(z)$. The parameters are set to $H=1$ and $\delta=2$.

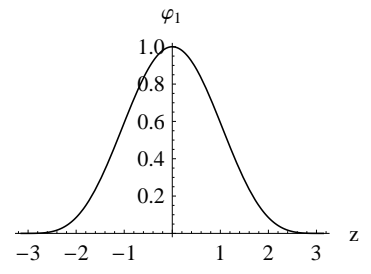

(a) $n=1$

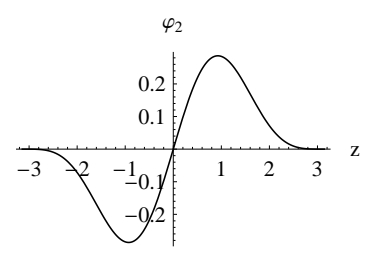

(b) $n=2$

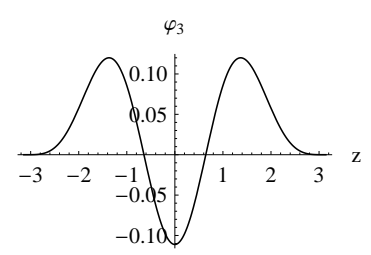

(c) $n=3$

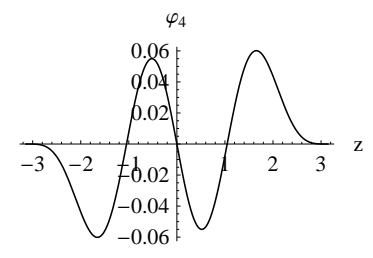

(d) $n=4$

FIG. 2: The shapes of the KK modes of the graviton $\varphi_{n}(z)$. The parameters are set to $H=1$ and $\delta=2$.

From the above discussion, $\mathrm{m}^{2}$ is always positive, and following the arguments given in Ref. [13], it can be shown that in the present case all the corresponding perturbation modes 
are stable. Now we need to further investigate the stability of this AdS brane, i.e., the scalar perturbation of the systems should be examined. The perturbed metric is given by [13]

$$
d s^{2}=\mathrm{e}^{2 A}\left[(1+2 \alpha) d z^{2}+(1+2 \beta) \hat{g}_{\mu \nu} d x^{\mu} d x^{\nu}\right] .
$$

As shown in Ref. [13], the following corresponding linearized five-dimensional Einstein-scalar equations can be obtained:

$$
\begin{aligned}
& \delta \phi=\frac{3}{\phi^{\prime}}\left(\alpha A^{\prime}-\beta^{\prime}\right), \quad \alpha+2 \beta=0, \\
& \beta^{\prime \prime}+\square \beta-\left(3 A^{\prime}+2 \frac{\phi^{\prime \prime}}{\phi^{\prime}}\right) \beta^{\prime} \\
& +\left(4 A^{\prime} \frac{\phi^{\prime \prime}}{\phi^{\prime}}-4 A^{\prime \prime}-6 H^{2}\right) \beta=0,
\end{aligned}
$$

where $\delta \phi$ denotes perturbations of the background scalar field. In order to examine the stability of this system, Eq. (24) is transformed into a form of the Schrödinger equation:

$$
\left[-\partial_{z}^{2}+V_{\mathrm{eff}}(z)\right] \omega\left(x^{\mu}, z\right)=\square \omega\left(x^{\mu}, z\right)
$$

where $\omega\left(x^{\mu}, z\right)$ and $V_{\text {eff }}(z)$ are defined as

$$
\begin{aligned}
\omega & \equiv \frac{1}{\phi^{\prime}(z)} \mathrm{e}^{\frac{3 A}{2}} \beta\left(x^{\mu}, z\right) \\
V_{\text {eff }} & \equiv-\frac{5}{2} A^{\prime \prime}+\frac{9}{4} A^{\prime 2}+A^{\prime} \frac{\phi^{\prime \prime}}{\phi^{\prime}}-\frac{\phi^{\prime \prime \prime}}{\phi^{\prime}}+2\left(\frac{\phi^{\prime \prime}}{\phi^{\prime}}\right)^{2}+6 H^{2} \\
& =\frac{H^{2}}{4 \delta^{2}}\left[\left(15 \delta^{2}-4 \delta-4\right)+3 \delta(3 \delta-2) \sec ^{2} \bar{z}\right]
\end{aligned}
$$

From above expression (27), it is clear that because the parameter $\delta$ satisfies $\delta>1$, the effective potential $V_{\text {eff }}\left(z \rightarrow \pm z_{b}\right) \rightarrow+\infty$, and has minimum $\frac{H^{2}}{2 \delta^{2}}(3 \delta-2)(4 \delta+1)$ (positive value) at $z=0$.

We decompose $\omega\left(x^{\mu}, z\right)$ in Eq. (25) as

$$
\omega\left(x^{\mu}, z\right)=f(z) X\left(x^{\mu}\right)
$$

and the following equations can be gotten

$$
\begin{aligned}
\square X\left(x^{\mu}\right) & =m^{2} X\left(x^{\mu}\right), \\
-f^{\prime \prime}(z)+V_{\mathrm{eff}}(z) f(z) & =m^{2} f(z)
\end{aligned}
$$


with $m$ the four-dimensional mass. It is known that Eq. (29) can be solved with suitable harmonic functions. There is the Breitenlohner-Freedman bound which allows the tachyonic mass to some extent from the condition of the normalization [13, 74]. The mass $m$ is bounded as

$$
m^{2} \geq-\frac{9}{4}
$$

which means that even when there are solutions with $-\frac{9}{4} \leq m^{2}<0$, such solutions are stable in spite of the tachyonic mass.

By substituting the effective potential (27) into Eq. (30), we can get

$$
\left[-\partial_{z}^{2}+\frac{3 H^{2}}{4 \delta}(3 \delta-2) \sec ^{2} \bar{z}\right] f_{n}(z)=E_{n}^{2} f_{n}(z),
$$

with $E_{n}^{2}=m_{n}^{2}-\frac{H^{2}}{4 \delta^{2}}\left(15 \delta^{2}-4 \delta-4\right)$. Since $\delta>1$, the solutions of the above Schrödinger Eq. (32) can be expressed as

$$
f_{n}(z) \propto \cos ^{\frac{3 \delta}{2}}(\bar{z}){ }_{2} \mathrm{~F}_{1}\left[-n, n+3 \delta, \frac{1+3 \delta}{2}, \frac{1-\sin \bar{z}}{2}\right],
$$

where $n=0,1,2, \cdots$. It is known that the corresponding perturbation modes tend to zero at the boundaries of the extra dimension, i.e., they are bound states, and all the scalar modes of the metric fluctuations can be localized on the AdS brane. The mass spectrum of the bound states is found to be

$$
E_{n}=\frac{H}{\delta}\left(n+\frac{3 \delta}{2}\right)
$$

or

$$
m_{n}=\frac{H}{\delta} \sqrt{n^{2}+6 \delta^{2}+(3 n-1) \delta-1} .
$$

It is clear that the mass of the ground state is $m_{0}=\frac{H}{\delta} \sqrt{6 \delta^{2}-\delta-1}$, and $m_{n}^{2} \geq \frac{H^{2}}{\delta^{2}}\left[n^{2}+\right.$ $\left.6 \delta^{2}+(3 n-1) \delta-1\right]>0$, so it is shown that the thick AdS brane is stable under the scalar perturbations.

\section{LOCALIZATION OF VARIOUS MATTERS ON THE THICK ANTI-DE SIT- TER BRANE}

In this section, we will investigate the character of the localization of the various bulk matter fields on the thick AdS brane. Spin-0 scalars, spin-1 vectors and spin-1/2 fermions 
will be considered by means of the gravitational interaction. Certainly, we have implicitly assumed that the various bulk matter fields considered below make little contribution to the bulk energy so that the solutions given in the previous section remain valid even in the presence of bulk matter fields. The mass spectra of the various matter fields on the thick brane will also be discussed by presenting the potential of the corresponding Schrödinger equation for the KK modes of the various matter fields.

\section{A. Spin-0 scalar fields}

We first consider the localization of real scalar fields on the thick brane obtained in the previous section, then turn to vectors and fermions in the next subsections. Let us start by considering the action of a massless real scalar coupled to gravity:

$$
S_{0}=-\frac{1}{2} \int d^{5} x \sqrt{-g} g^{M N} \partial_{M} \Phi \partial_{N} \Phi
$$

Using the metric (21), the equation of motion derived from (36) reads as

$$
\frac{1}{\sqrt{-\hat{g}}} \partial_{\mu}\left(\sqrt{-\hat{g}} \hat{g}^{\mu \nu} \partial_{\nu} \Phi\right)+e^{-3 A} \partial_{z}\left(e^{3 A} \partial_{z} \Phi\right)=0 .
$$

Then, by using of the KK decomposition

$$
\Phi(x, z)=\sum_{n} \phi_{n}(x) \chi_{n}(z) e^{-3 A / 2}
$$

the four-dimensional scalar fields $\phi_{n}(x)$ should satisfy the four-dimensional massive KleinGordon equation:

$$
\left(\frac{1}{\sqrt{-\hat{g}}} \partial_{\mu}\left(\sqrt{-\hat{g}} \hat{g}^{\mu \nu} \partial_{\nu}\right)-m_{n}^{2}\right) \phi_{n}(x)=0 .
$$

Therefore the equation for the scalar KK modes $\chi_{n}(z)$ can be expressed as

$$
\left[-\partial_{z}^{2}+V_{0}(z)\right] \chi_{n}(z)=m_{n}^{2} \chi_{n}(z)
$$

which is a Schrödinger equation with the effective potential given by

$$
V_{0}(z)=\frac{3}{2} A^{\prime \prime}+\frac{9}{4} A^{\prime 2}
$$

where $m_{n}$ is the mass of the KK excitation. It is clear that the potential $V_{0}(z)$ defined in (41) is a four-dimensional mass-independent potential. 
The full five-dimensional action (36) can be reduced to one four-dimensional action for a massless scalar field plus an infinite sum of massive scalar actions in four-dimension

$$
S_{0}=-\frac{1}{2} \sum_{n} \int d^{4} x \sqrt{-\hat{g}}\left(\hat{g}^{\mu \nu} \partial_{\mu} \phi_{n} \partial_{\nu} \phi_{n}+m_{n}^{2} \phi_{n}^{2}\right)
$$

when integrated over the extra dimension, in which it is required that Eq. (40) is satisfied and the following orthonormality conditions are obeyed:

$$
\int_{-z_{b}}^{+z_{b}} \chi_{m}(z) \chi_{n}(z) d z=\delta_{m n}
$$

For the thick AdS brane, because the effective potential for the scalar KK modes (41) is the same as the potential for the gravity (17), the localization of scalars is the same as the situation of gravity. Hence, the four-dimensional massless scalar (the zero mode) is not localized on the thick AdS brane for $\delta>1$. And all the KK modes are massive bound states and are localized on the brane. The mass spectrum of the KK modes is discrete.

\section{B. Spin-1 vector fields}

We now turn to spin-1 vector fields. We begin with the five-dimensional action of a vector field

$$
S_{1}=-\frac{1}{4} \int d^{5} x \sqrt{-g} g^{M N} g^{R S} F_{M R} F_{N S},
$$

where $F_{M N}=\partial_{M} A_{N}-\partial_{N} A_{M}$ is the field tensor as usual. From this action the equations of motion are derived as follows

$$
\frac{1}{\sqrt{-g}} \partial_{M}\left(\sqrt{-g} g^{M N} g^{R S} F_{N S}\right)=0 .
$$

By using the background metric (2), the equations of motion read as

$$
\begin{array}{r}
\frac{1}{\sqrt{-\hat{g}}} \partial_{\nu}\left(\sqrt{-\hat{g}} \hat{g}^{\nu \rho} \hat{g}^{\mu \lambda} F_{\rho \lambda}\right)+\hat{g}^{\mu \lambda} e^{-A} \partial_{z}\left(e^{A} F_{4 \lambda}\right)=0, \\
\partial_{\mu}\left(\sqrt{-\hat{g}} \hat{g}^{\mu \nu} F_{\nu 4}\right)=0 .
\end{array}
$$

Because the fourth component $A_{4}$ has no zero mode in the effective four-dimensional theory, we assume that it is $Z_{2}$-odd with respect to the extra dimension $z$. Furthermore, in order to be consistent with the gauge invariant equation $\oint d z A_{4}=0$, we choose $A_{4}=0$ by using gauge freedom. Then, the action (44) can be reduced to

$$
S_{1}=-\frac{1}{4} \int d^{5} x \sqrt{-g}\left[F_{\mu \nu} F^{\mu \nu}+2 e^{-A} g^{\mu \nu} \partial_{z} A_{\mu} \partial_{z} A_{\nu}\right]
$$


With the decomposition of the vector field

$$
A_{\mu}(x, z)=\sum_{n} a_{\mu}^{(n)}(x) \rho_{n}(z) e^{-A / 2}
$$

and the orthonormality condition

$$
\int_{-z_{b}}^{+z_{b}} \rho_{m}(z) \rho_{n}(z) d z=\delta_{m n}
$$

the action (48) can be simplified as

$$
\begin{aligned}
S_{1}=\sum_{n} \int d^{4} x \sqrt{-\hat{g}}[ & -\frac{1}{4} \hat{g}^{\mu \alpha} \hat{g}^{\nu \beta} f_{\mu \nu}^{(n)} f_{\alpha \beta}^{(n)} \\
& \left.-\frac{1}{2} m_{n}^{2} \hat{g}^{\mu \nu} a_{\mu}^{(n)} a_{\nu}^{(n)}\right],
\end{aligned}
$$

where $f_{\mu \nu}^{(n)}=\partial_{\mu} a_{\nu}^{(n)}-\partial_{\nu} a_{\mu}^{(n)}$ is the four-dimensional field strength tensor. Therefore we have obtained a four-dimensional theory of a gauge particle (massless) and infinite towers of massive vector fields. In the above process, it has been required that the vector KK modes $\rho_{n}(z)$ should satisfy the following Schrödinger equation

$$
\left[-\partial_{z}^{2}+V_{1}(z)\right] \rho_{n}(z)=m_{n}^{2} \rho_{n}(z)
$$

with the mass-independent potential $V_{1}(z)$ given by

$$
V_{1}(z)=\frac{H^{2}}{4 \delta}\left[(2+\delta) \sec ^{2} \bar{z}-\delta\right]
$$

The potential also has a minimum $\frac{H^{2}}{2 \delta}$ at $z=0$ and tends to positive infinity at $z=z_{b}$ for $\delta>1$. The shapes of the potential $V_{1}(z)$ are plotted in Fig. 3. The potential $V_{1}(z)$ are always positive, so the zero mode is not localized on the AdS brane. However, the massive modes can be localized on the brane. Eq. (152) with this potential can be turned into the following Schrödinger equation:

$$
\left[-\partial_{z}^{2}+\frac{H^{2}}{4 \delta}(2+\delta) \sec ^{2} \bar{z}\right] \rho_{n}(z)=E_{n}^{2} \rho_{n}(z)
$$

with $E_{n}^{2}=m_{n}^{2}+\frac{H^{2}}{4}$. The solutions of Eq. (154) for $\delta>1$ can be expressed as follows

$$
\rho_{n} \propto \cos ^{1+\frac{\delta}{2}}(\bar{z}){ }_{2} \mathrm{~F}_{1}\left[1-n, 1+n+\delta, \frac{3+\delta}{2}, \frac{1-\sin \bar{z}}{2}\right],
$$

where $n=1,2, \cdots$, and the discrete mass spectrum can be written as

$$
m_{n}=\frac{H}{\delta} \sqrt{n(n+\delta)},
$$


so all the KK modes are bound states and can also be trapped on the brane. We can find that the ground state is a massive state with $m_{1}=\frac{H}{\delta} \sqrt{1+\delta}$. The shapes of the KK modes are plotted in Fig. 4 ,

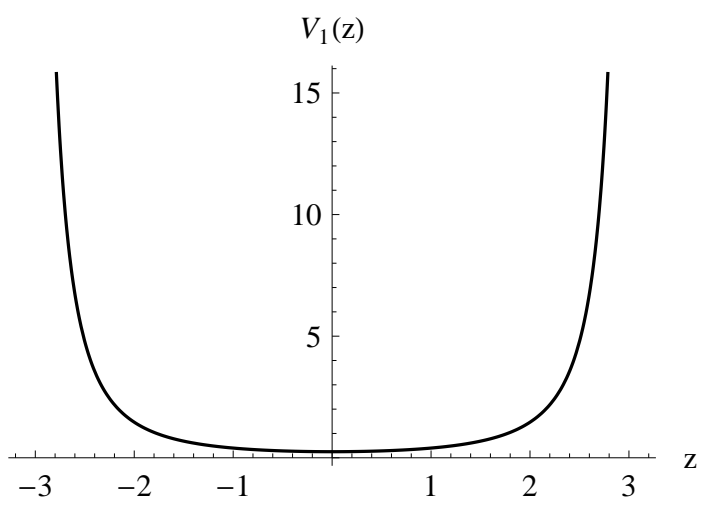

FIG. 3: The shape of the potential of the vector field $V_{1}(z)$. The parameters are set to $H=1$ and $\delta=2$.

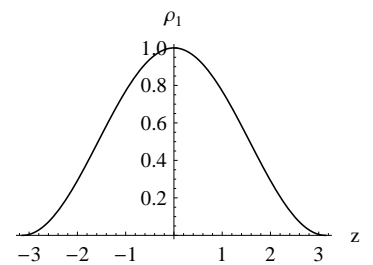

(a) $n=1$

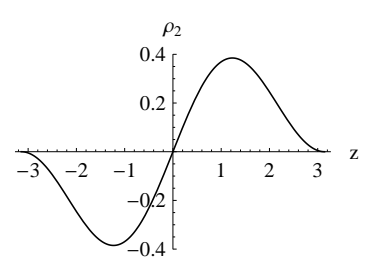

(b) $n=2$

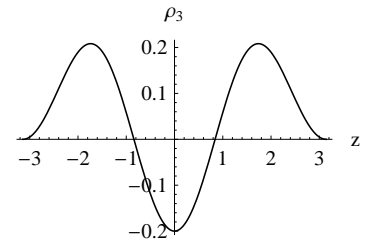

(c) $n=3$

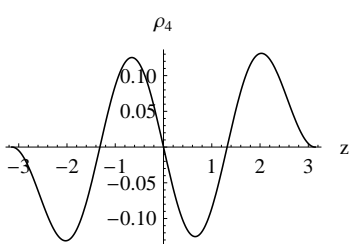

(d) $n=4$

FIG. 4: The shapes of the KK modes of the vector fields $\rho_{n}(z)$. The parameters are set to $H=1$, and $\delta=2$.

\section{Spin-1/2 fermion fields}

Finally, we will study the localization of fermions on the thick AdS brane. In fivedimensional space-time, fermions are four component spinors and their Dirac structure can be described by the curved space gamma matrices $\Gamma^{M}=e^{-A}\left(\gamma^{\mu}, \gamma^{5}\right)$, where $\gamma^{\mu}$ and $\gamma^{5}$ are the usual flat gamma matrices in the four-dimensional Dirac representation. The Dirac action of a massless spin-1/2 fermion coupled to the background scalar $\phi(6)$ is

$$
S_{1 / 2}=\int d^{5} x \sqrt{-g}\left[\bar{\Psi} \Gamma^{M}\left(\partial_{M}+\omega_{M}\right) \Psi-\eta \bar{\Psi} F(\phi) \Psi\right]
$$


where $\eta$ is a coupling constant. The nonvanishing components of the spin connection $\omega_{M}$ for the background metric (2) are

$$
\omega_{\mu}=\frac{1}{2} A^{\prime} \gamma_{\mu} \gamma_{5}+\hat{\omega}_{\mu}
$$

with $\hat{\omega}_{\mu}$ the spin connection derived from the metric $\hat{g}_{\mu \nu}(x)$. Then the equation of motion is given by

$$
\left[\gamma^{\mu}\left(\partial_{\mu}+\hat{\omega}_{\mu}\right)+\gamma^{5}\left(\partial_{z}+2 A^{\prime}\right)-\eta \mathrm{e}^{A} F(\phi)\right] \Psi=0
$$

where $\gamma^{\mu}\left(\partial_{\mu}+\hat{\omega}_{\mu}\right)$ is the Dirac operator on the AdS brane.

Now we would like to investigate the localization of the Dirac spinor on the AdS brane by studying the above five-dimensional Dirac equation. Because of the Dirac structure of the fifth gamma matrix $\gamma^{5}$, we expect that the left- and right-chiral projections of the fourdimensional part have different behaviors. From the equation of motion (59), we will search for the solutions of the general chiral decomposition

$$
\Psi=\sum_{n}\left[\psi_{L, n}(x) L_{n}(z)+\psi_{R, n}(x) R_{n}(z)\right] \mathrm{e}^{-2 A},
$$

where $\psi_{L}=\frac{1-\gamma^{5}}{2} \psi$ and $\psi_{R}=\frac{1+\gamma^{5}}{2} \psi$ are the left- and right-chiral components of a fourdimensional Dirac field $\psi$, respectively. By demanding $\psi_{L, R}$ satisfy the four-dimensional massive Dirac equations $\gamma^{\mu}\left(\partial_{\mu}+\hat{\omega}_{\mu}\right) \psi_{L, R}=m \psi_{R, L}$, we obtain the following coupled equations for the fermion KK modes $L_{n}$ and $R_{n}$ :

$$
\begin{aligned}
{\left[\partial_{z}+\eta \mathrm{e}^{A} F(\phi)\right] L_{n}(z) } & =m R_{n}(z), \\
{\left[\partial_{z}-\eta \mathrm{e}^{A} F(\phi)\right] R_{n}(z) } & =-m L_{n}(z) .
\end{aligned}
$$

From the above equations, we can obtain the Schrödinger-like equations for the KK modes of the left- and right-chiral fermions:

$$
\begin{aligned}
& \left(-\partial_{z}^{2}+V_{L}(z)\right) L_{n}=m^{2} L_{n} \\
& \left(-\partial_{z}^{2}+V_{R}(z)\right) R_{n}=m^{2} R_{n}
\end{aligned}
$$

where the effective potentials of Eq. (62) are given by

$$
\begin{aligned}
V_{L}(z) & =\left(\eta \mathrm{e}^{A} F(\phi)\right)^{2}-\partial_{z}\left(\eta \mathrm{e}^{A} F(\phi)\right), \\
V_{R}(z) & =\left.V_{L}(z)\right|_{\eta \rightarrow-\eta} .
\end{aligned}
$$


For the purpose of getting the standard four-dimensional action for a massless fermion and an infinite sum of the massive fermions,

$$
\begin{aligned}
S_{\frac{1}{2}} & =\int d^{5} x \sqrt{-g} \bar{\Psi}\left[\Gamma^{M}\left(\partial_{M}+\omega_{M}\right)-\eta F(\phi)\right] \Psi \\
& =\sum_{n} \int d^{4} x \sqrt{-\hat{g}} \bar{\psi}_{n}\left[\gamma^{\mu}\left(\partial_{\mu}+\hat{\omega}_{\mu}\right)-m_{n}\right] \psi_{n},
\end{aligned}
$$

we need the following orthonormality conditions for $L_{n}$ and $R_{n}$ :

$$
\begin{gathered}
\int_{-z_{b}}^{z_{b}} L_{m} L_{n} d z=\delta_{m n} \\
\int_{-z_{b}}^{z_{b}} R_{m} R_{n} d z=\delta_{m n} \\
\int_{-z_{b}}^{z_{b}} L_{m} R_{n} d z=0
\end{gathered}
$$

From Eqs. (62) and (63), we can see that, in order to localize the left- and right-chiral fermions, there must be some kind of scalar-fermion coupling. This situation is similar to the one in Refs. [35, 48], in which the authors introduced the mass term $m \epsilon(z) \bar{\Psi} \Psi$ for the localization of the fermion fields on a brane. For the thick branes arising from a real scalar field $\phi$, the scalar-fermion coupling such as $\eta \bar{\Psi} \phi \Psi, \eta \bar{\Psi} \phi^{k} \Psi, \eta \bar{\Psi} \tan ^{1 / s}(\phi) \Psi$, etc., can be introduced for fermion localization [22, 50, 53, 60]. For the thick brane generated by two scalar $\phi$ and $\chi$, in order to localize the fermions, the coupling terms $\eta \bar{\Psi} \phi \chi \Psi$ and $\eta \bar{\Psi} \phi \Psi+\eta^{\prime} \bar{\Psi} \chi \Psi$ were introduced in Ref. [70]. Moreover, if we demand that $V_{L}(z)$ and $V_{R}(z)$ are $Z_{2}$-even with respect to the extra dimension $z, F(\phi)$ should be an odd function of the "kink" $\phi(z)$. In this paper, we choose the simplest Yukawa coupling: $F(\phi)=\phi$. Then the explicit forms of the potentials $(\underline{63})$ are

$$
\begin{aligned}
V_{L}(z)= & \eta^{2} \phi_{0}^{2} \cos ^{-2 \delta}(\bar{z}) \operatorname{arcsinh}^{2}(\tan \bar{z}) \\
& -\frac{\eta H \phi_{0}}{\delta \cos ^{1+\delta}(\bar{z})}[\delta \sin \bar{z} \operatorname{arcsinh}(\tan \bar{z})+1], \\
V_{R}(z)= & \left.V_{L}(z)\right|_{\eta \rightarrow-\eta} .
\end{aligned}
$$

At the location of the AdS brane, both of the potentials for the left- and right-chiral fermions have minimum $V_{L}(z=0)=-\eta H \phi_{0} / \delta$ and $V_{R}(z=0)=\eta H \phi_{0} / \delta$, and the potentials $V_{L, R}\left(z \rightarrow \pm z_{b}\right) \rightarrow+\infty$ as shown in Fig. 5 .

From Fig. 5, only the potential of the left-chiral fermions has negative value at the location of the thick brane for the positive coupling constant $\eta$, so only the left-chiral fermion zero 


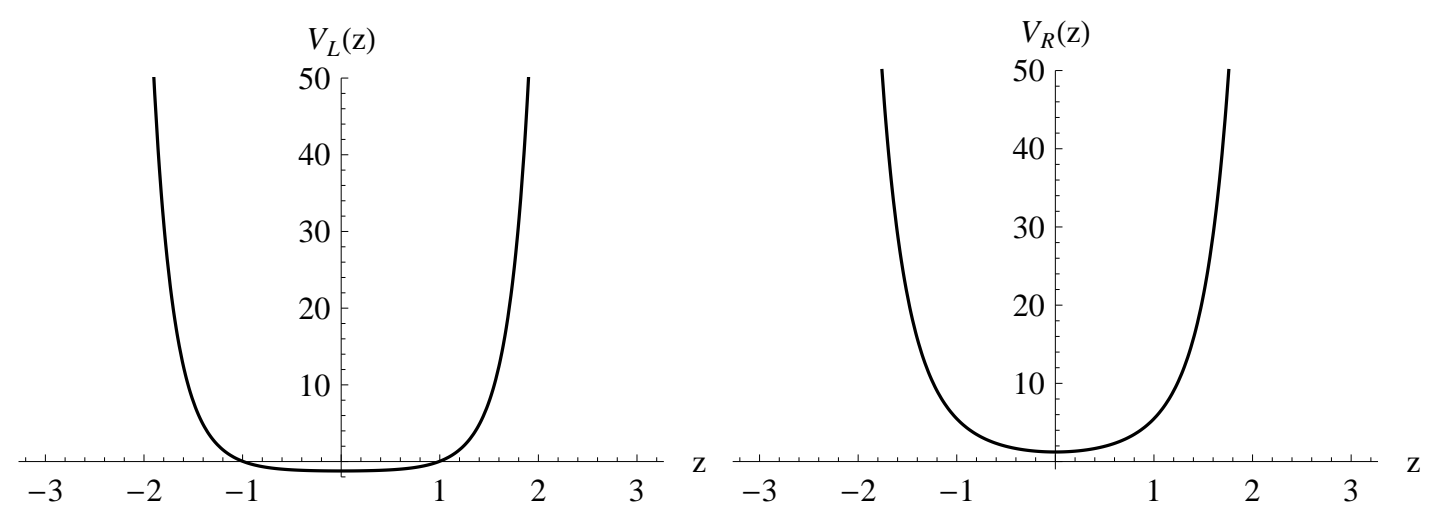
(a) $V_{L}(z)$
(b) $V_{R}(z)$

FIG. 5: The shapes of the potentials of the left- and right-chiral fermion KK modes. The parameters are set to $\delta=2, H=1$ and $\eta=1$.

mode can be localized on the brane. By setting $m=0$ in Eq. (61), the left-chiral fermion zero mode can be obtained:

$$
L_{0}(z) \propto \exp [-\eta I(z)]
$$

where the exponential factor $I(z)$ can be expressed as

$$
\begin{aligned}
I(z)= & \int_{0}^{z} d z^{\prime} \mathrm{e}^{A\left(z^{\prime}\right)} \phi\left(z^{\prime}\right) \\
= & \frac{2^{1-\delta} \phi_{0} \delta}{H(\delta-1)^{2}}\left\{{ }_{3} \mathrm{~F}_{2}\left(1-\delta, \frac{1-\delta}{2}, \frac{1-\delta}{2} ; \frac{3-\delta}{2}, \frac{3-\delta}{2} ;-1\right)\right. \\
& -\varrho^{1-\delta}(z)\left[{ }_{3} \mathrm{~F}_{2}\left(1-\delta, \frac{1-\delta}{2}, \frac{1-\delta}{2} ; \frac{3-\delta}{2}, \frac{3-\delta}{2} ;-\varrho^{2}(z)\right)\right. \\
& \left.\left.+(\delta-1){ }_{2} \mathrm{~F}_{1}\left(\frac{1-\delta}{2}, 1-\delta, \frac{3-\delta}{2},-\varrho^{2}(z)\right) \ln (\varrho(z))\right]\right\}
\end{aligned}
$$

with $\varrho(z)=\sec \bar{z}+\tan \bar{z}$. The curve for the exponential factor is plotted in Fig. 6(a), The exponential factor $I(z)$ tends to positive infinity, so the left-chiral fermion zero mode $L_{0} \rightarrow 0$ as $z \rightarrow \pm z_{b}$, and is localized on the AdS brane, which is shown in Fig. 6(b),

For the massive KK modes of the left- and right-chiral fermions, Eqs. (62a) and (62b) cannot be analytically solved. But we can solve them by numerical method. The KK modes

of the left- and right-chiral fermions are plotted in Figs. 7 and 8 , respectively. All the KK modes for both the left- and right-chiral fermions are bound states. The discrete mass 


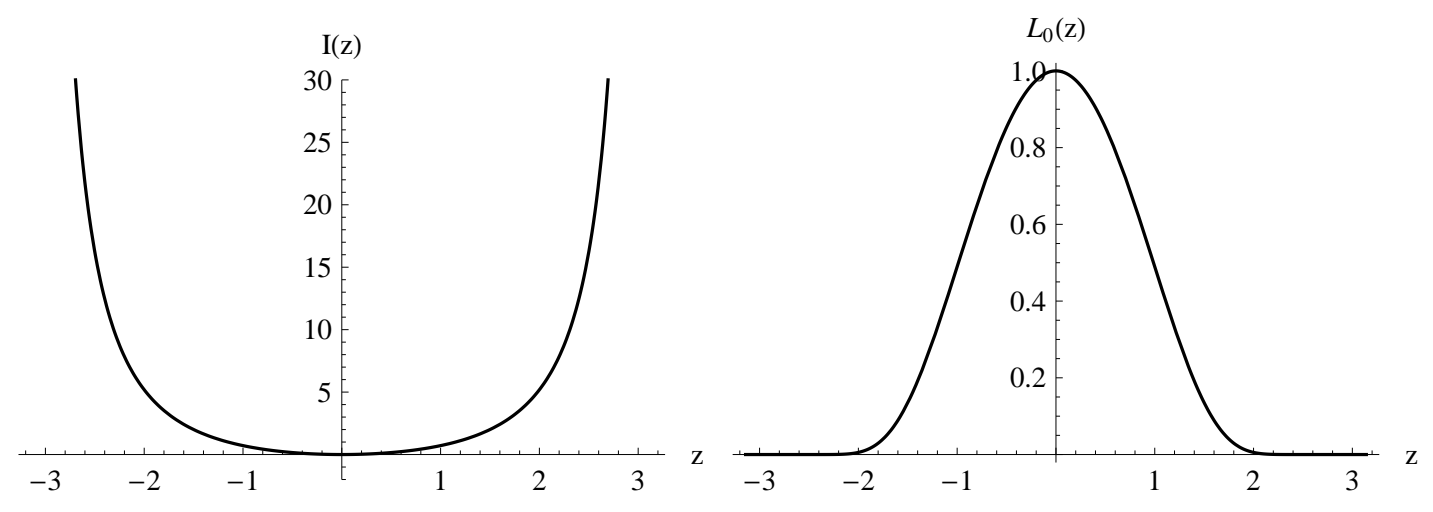
(a) $I(z)$
(b) $L_{0}$

FIG. 6: The shape of the exponential factor $I(z)$ and the left-chiral fermion zero mode $L_{0}$. The parameters are set to $H=1, \eta=1$ and $\delta=2$.

spectra for the KK modes are calculated as follows:

$$
\begin{gathered}
m_{L_{n}}=\{0,1.78,2.76,3.63,4.45,5.22,5.98,6.71, \\
7.43,8.14,8.84, \cdots\}, \\
m_{R_{n}}=\left\{\begin{array}{l}
1.78,2.76,3.63,4.45,5.22,5.98,6.71, \\
7.43,8.14,8.84, \cdots\},
\end{array}\right.
\end{gathered}
$$

where the parameters are set to $H=1, \eta=1$ and $\delta=2$. Hence the ground state of the left-chiral fermions is the massless mode; however, the ground state of the right-chiral fermions is a massive mode. The mass spectra are also shown in Fig. 9, So we can obtain the four-dimensional massless left-chiral fermion and the massive Dirac fermions consisting of the pairs of the left- and right-chiral KK modes coupling together through mass terms.

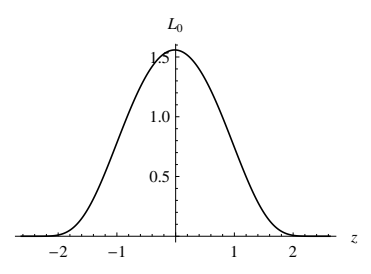

(a) $n=0$

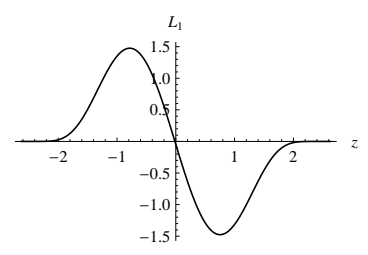

(b) $n=1$

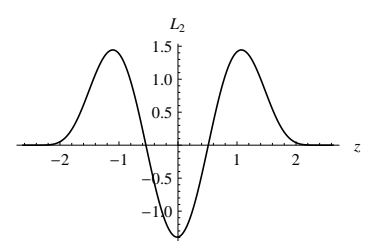

(c) $n=2$

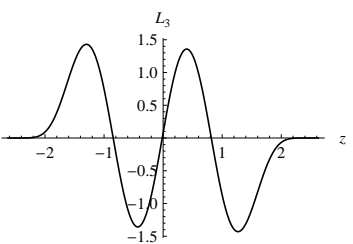

(d) $n=3$

FIG. 7: The shapes of the KK modes of the left-chiral fermions. The parameters are set to $H=1$, $\eta=1$ and $\delta=2$. 


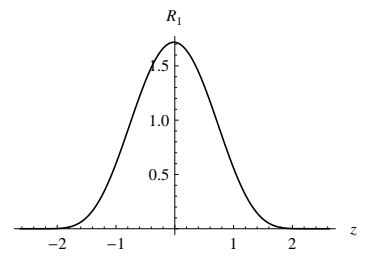

(a) $n=1$

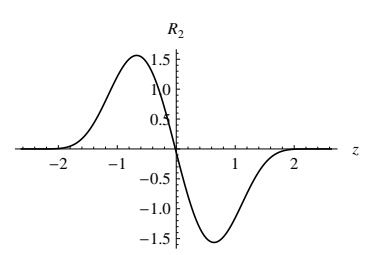

(b) $n=2$

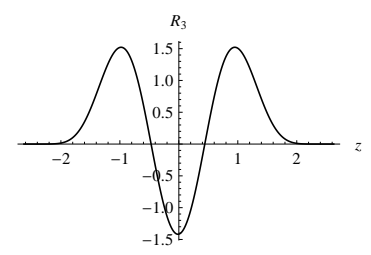

(c) $n=3$

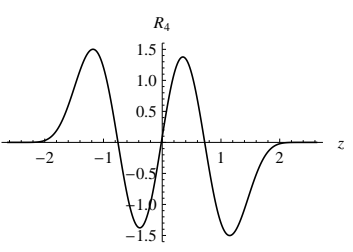

(d) $n=4$

FIG. 8: The shapes of the KK modes of the right-chiral fermions. The parameters are set to $H=1$, $\eta=1$ and $\delta=2$.

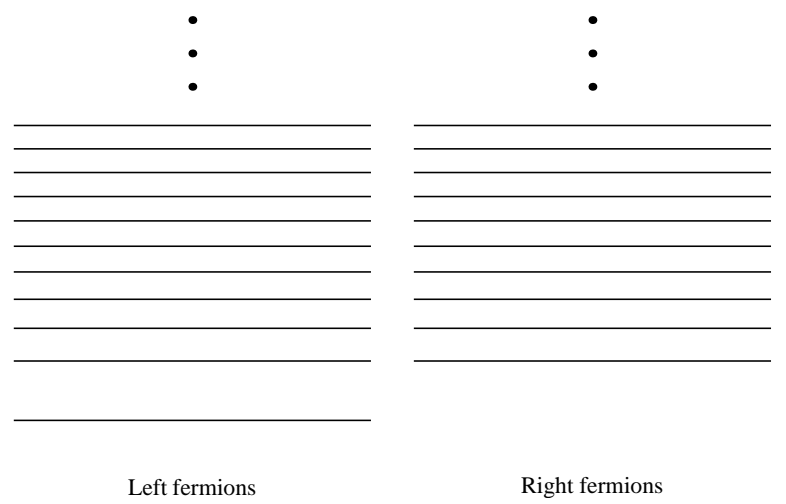

FIG. 9: The $m_{L_{n}, R_{n}}$ spectra of the left- and right-chiral fermions. The parameters are set to $H=1$, $\eta=1$ and $\delta=2$.

\section{CONCLUSION AND DISCUSSION}

In this paper, we first reviewed a thick AdS brane embedded in five-dimensional spacetime, in which the behavior of the warp factor is related to a parameter $\delta$. For $\delta<0$, the warp factor tends to zero at the boundaries of the extra dimension, while the energy density has no lump at $z \approx 0$, which cannot be considered as a thick brane. For $\delta>1$, the warp factor tends to infinity at the boundaries, and the energy density has a lump at $z \approx 0$, which indicates that the AdS thick brane locates at $z \approx 0$. Hence, we only consider the case of $\delta>1$. Then we studied the mass-independent potentials of the KK modes for gravity and various spin fields in the corresponding Schrödinger equations. In this way, the localization and mass spectra of gravity and various matters with spin-0, 1 and $1 / 2$ on this kind of AdS brane were investigated.

For gravity, the potential of the KK modes in the corresponding Schrödinger equation is divergent when far away from the brane. Such potential suggests that the mass spectrum of 
the gravity KK modes consists of an infinite number of discrete bound states. Although, the zero mode of gravity is not localized on the AdS brane, the massive modes can be localized on the brane. The scalar perturbations of the thick AdS brane has been analyzed, and as a result, it is shown that the brane is stable under the scalar perturbations.

For spin-0 scalars and spin-1 vectors, the character of localization is similar to the case of gravity; i.e., all the scalar and vector KK modes are bound states, and the zero modes are not localized on the brane.

For spin-1/2 fermions, in order to localize the left- and right-chiral fermions, we introduced the usual Yukawa coupling $\eta \bar{\Psi} \phi \Psi$ with a positive coupling constant $\eta$. Both potentials

have the same asymptotic behavior: $V_{L, R}\left(z \rightarrow \pm z_{b}\right) \rightarrow+\infty$, and only the potential of the left-chiral fermion KK modes has a finite negative well at $z=0$. So only the left-chiral fermion zero mode could be localized on the brane; i.e., there exists only the four-dimensional massless left-chiral fermion. And both the left- and right-chiral fermion KK modes have an infinite number of bound states. Since a pair of left- and right-chiral KK modes couple together through a mass term to become a four-dimensional Dirac fermion, a series of fourdimensional Dirac fermions with a discrete mass spectrum could be obtained on the AdS branes.

\section{Acknowledgement}

We are grateful to the referee, whose comments led to the improvement of this paper. This work was supported by the Program for New Century Excellent Talents in University, the Huo Ying-Dong Education Foundation of Chinese Ministry of Education (No. 121106), the National Natural Science Foundation of China (No. 11075065), the Doctoral Program Foundation of Institutions of Higher Education of China (No. 20090211110028), the Key Project of Chinese Ministry of Education (No. 109153), and the Natural Science Foundation of Gansu Province, China (No. 096RJZA055).

[1] V.A. Rubakov and M.E. Shaposhnikov, Phys. Lett. B 125 (1983) 136; V.A. Rubakov and M.E. Shaposhnikov, Phys. Lett. B 125 (1983) 139; E.J. Squires, Phys. Lett. B 167 (1986) 286. 
[2] M. Visser, Phys. Lett. B 159 (1985) 22.

[3] N. Arkani-Hamed, S. Dimopoulos and G. Dvali, Phys. Lett. B 429 (1998) 263; I. Antoniadis, N. Arkani-Hamed, S. Dimopoulos and G. Dvali, Phys. Lett. B 436 (1998) 257; N. ArkaniHamed, S. Dimopoulos, G. Dvali and N. Kaloper, Phys. Rev. Lett. 84 (2000) 586.

[4] S. Randjbar-Daemi and C. Wetterich, Phys. Lett. B 166 (1986) 65.

[5] L. Randall and R. Sundrum, Phys. Rev. Lett. 83 (1999) 3370; Phys. Rev. Lett. 83 (1999) 4690.

[6] J. Lykken and L. Randall, JHEP 0006 (2000) 014.

[7] I. Antoniadis, Phys. Lett. B 246 (1990) 377.

[8] N. Arkani-Hamed, S. Dimopoulos, N. Kaloper and R. Sundtrum, Phys. Lett. B 480 (2000) 193; S. Kachru, M. Schulz and E. Silverstein, Phys. Rev. D 62 (2000) 045021; A. Kehagias, Phys. Lett. B 600 (2004) 133.

[9] O. DeWolfe, D.Z. Freedman, S.S. Gubser and A. Karch, Phys. Rev. D 62 (2000) 046008.

[10] D. Stojkovic, Phys. Rev. D 63 (2000) 025010.

[11] M. Gremm, Phys. Lett. B 478 (2000) 434; Phys. Rev. D 62 (2000) 044017;

[12] K. Ghoroku and M. Yahiro, hep-th/0305150; A. Kehagias and K. Tamvakis, Mod. Phys. Lett. A 17 (2002) 1767; Phys. Lett. B 504(2001) 38; M. Giovannini, Phys. Rev. D 64 (2001) 064023; Phys. Rev. D 65 (2002) 064008;

[13] S. Kobayashi, K. Koyama and J. Soda, Phys. Rev. D 65 (2002) 064014.

[14] C. Csaki, J. Erlich, T. Hollowood and Y. Shirman, Nucl. Phys. B 581 (2000) 309.

[15] G. Dvali, G. Gabadadze and M. Porrati, Phys. Lett. B 485 (2000) 208.

[16] R. Gregory, V.A. Rubakov and S.M. Sibiryakov, Phys. Rev. Lett. 84, (2000) 5928.

[17] A. Campos, Phys. Rev. Lett. 88 (2002) 141602.

[18] A. Wang, Phys. Rev. D 66(2002) 024024.

[19] R. Emparan, R. Gregory and C. Santos, Phys. Rev. D 63 (2001) 104022; R. Guerrero, A. Melfo and N. Pantoja, Phys. Rev. D 65 (2002) 125010; A. Melfo, N. Pantoja and A. Skirzewski, Phys. Rev. D 67 (2003) 105003; K.A. Bronnikov and B.E. Meierovich, Grav. Cosmol. 9 (2003) 313; O. Castillo-Felisola, A. Melfo, N. Pantoja and A. Ramirez, Phys. Rev. D 70 (2004) 104029.

[20] V. Dzhunushaliev, V. Folomeev, D. Singleton and S. Aguilar-Rudametkin, Phys. Rev. D 77 (2008) 044006; V. Dzhunushaliev, V. Folomeev, K. Myrzakulov and R. Myrzakulov, Gen. Rel. Grav. 41 (2008) 131; D. Bazeia, F.A. Brito and J.R. Nascimento, Phys. Rev. D 68 (2003) 085007; D. Bazeia, F.A. Brito and A.R. Gomes, JHEP 0411 (2004) 070; D. Bazeia and A.R. Gomes, JHEP 0405 (2004) 012; D. Bazeia, A.R. Gomes and L. Losano, Int. J. Mod. Phys. A 24 (2009) 1135. 
[21] D. Bazeia, F.A. Brito and L. Losano, JHEP 0611 (2006) 064; V.I. Afonso, D. Bazeia and L. Losano, Phys. Lett. B634 (2006) 526.

[22] T.R. Slatyer and R.R. Volkas, JHEP 0704 (2007) 062; R. Davies, D.P. George and R.R. Volkas, Phys. Rev. D 77 (2008) 124038.

[23] T. Gherghetta and M. Shaposhnikov, Phys. Rev. Lett. 85 (2000) 240.

[24] I.P. Neupane, JHEP 0009 (2000) 040; I.P. Neupane, Class. Quant. Grav. 19 (2002) 5507.

[25] O. Arias, R. Cardenas and I. Quiros, Nucl. Phys. B 643 (2002) 187; N. Barbosa-Cendejas and A. Herrera-Aguilar, JHEP 0510 (2005) 101; N. Barbosa-Cendejas and A. Herrera-Aguilar, Phys. Rev. D 73 (2006) 084022.

[26] M. Cvetic, S. Griffies and S.-J. Rey, Nucl. Phys. B381 (1992) 301; M. Cvetic and H.H. Soleng, Phys. Rept. 282 (1997) 159; M. Cvetic and M. Robnik, Phys. Rev. D 77 (2008) 124003.

[27] N. Barbosa-Cendejas, A. Herrera-Aguilar, M.A. Reyes Santos and C. Schubert, Phys. Rev. D 77 (2008) 126013.

[28] N. Barbosa-Cendejas, A. Herrera-Aguilar, U. Nucamendi and I. Quiros, arXiv:0712.3098[hepth].

[29] S. Kobayashi, K. Koyama and J. Soda, Phys. Rev. D 65 (2002) 064014.

[30] A. Karch and L. Randall, JHEP 0105 (2001) 008.

[31] A. Herrera-Aguilar, D. Malagon-Morejon, R.R. Mora-Luna and U. Nucamendi, Mod. Phys. Lett. A25 (2010) 2089.

[32] Y.-X. Liu, K. Yang and Y. Zhong JHEP 1010 (2010) 069.

[33] A. Brandhuber and K. Sfetsos, JHEP 9910 (1999) 013.

[34] J.I. Diaz, J. Negro, L.M. Nieto, O. Rosas-Ortiz, J. Phys. A: Math. Gen. 32 (1999) 8447.

[35] I. Oda, Phys. Rev. D 64 (2001) 026002.

[36] Y.-X. Liu, Y. Zhong and K. Yang, Europhys. Lett. 90 (2010) 51001.

[37] A. Balcerzak and M.P. Dạbrowski, Phys. Rev. D 81 (2010) 123527.

[38] Y. Zhong, Y.-X. Liu and K. Yang, Phys. Lett. B 699, 398 (2011).

[39] M. Gogberashvili and D. Singleton, Phys. Rev. D 69 (2004) 026004; M. Gogberashvili and D. Singleton, Phys. Lett. B582 (2004) 95.

[40] H. Guo, Y.-X. Liu, S.-W. Wei and C.-E. Fu, arXiv:1008.3686[hep-th].

[41] A. Herrera-Aguilar, D. Malagon-Morejon and R.R. Mora-Luna, JHEP 1011 (2010) 015.

[42] S. SenGupta, arXiv:0812.1092[hep-th].

[43] V. Dzhunushaliev, V. Folomeev, and M. Minamitsuji, Rept. Prog. Phys. 73 (2010) 066901.

[44] M. Shifman, Int. J. Mod. Phys. A25 (2010) 199.

[45] T.G. Rizzo, in Gravitational Physics: Testing Gravity from Submillimetre to Cosmic: Proceedings of the VIII Mexican School on Gravitation and Mathematical Physics, edited by H. 
A. Morales-Tecotl, L. A. Urena-Lopez, R. Linares-Romero, and H. H. Garcia-Compean, AIP Conf. Proc. 1256 (AIP, New York, 2010). p27-50.

[46] P.D. Mannheim, Brane-localized gravity, World Scientific Publishing Company, Singapore (2005).

[47] R. Maartens and K. Koyama, Living Rev. Rel. 13 (2010) 5.

[48] B. Bajc and G. Gabadadze, Phys. Lett. B 474 (2000) 282.

[49] I. Oda, Phys. Lett. B 496 (2000) 113.

[50] Y.-X. Liu, X.-H. Zhang, L.-D. Zhang and Y.-S. Duan, JHEP 0802 (2008) 067; Y.-X. Liu, L.-D. Zhang, L.-J. Zhang and Y.-S. Duan, Phys. Rev. D 78, 065025 (2008).

[51] Y. Grossman and N. Neubert, Phys. Lett. B 474 (2000) 361; R. Koley and S. Kar, Phys. Lett. B 623 (2005) 244; A. Melfo, N. Pantoja and J.D. Tempo, Phys. Rev. D 73 (2006) 044033.

[52] S. Ichinose, Phys. Rev. D 66 (2002) 104015.

[53] C. Ringeval, P. Peter and J.P. Uzan, Phys. Rev. D 65 (2002) 044016.

[54] S. Randjbar-Daemi and M. Shaposhnikov, Phys. Lett. B 492 (2000) 361.

[55] R. Koley and S. Kar, Class. Quant. Grav. 22 (2005) 753.

[56] S.L. Dubovsky, V.A. Rubakov and P.G. Tinyakov, Phys. Rev. D 62 (2000) 105011.

[57] Y. Brihaye and T. Delsate, Phys. Rev. D 78 (2008) 025014.

[58] Y.-X. Liu, L. Zhao and Y.-S. Duan, JHEP 0704 (2007) 097; Y.-X. Liu, L. Zhao, X.-H. Zhang and Y.-S. Duan, Nucl. Phys. B 785 (2007) 234; Y.-Q. Wang, T.-Y. Si, Y.-X. Liu and Y.-S. Duan, Mod. Phys. Lett. A 20 (2005) 3045; L. Zhao, Y.-X. Liu and Y.-S. Duan, Mod. Phys. Lett. A 23 (2008) 1129.

[59] C.A.S. Almeida, R. Casana, M.M. Ferreira and A.R. Gomes, Phys. Rev. D 79 (2009) 125022.

[60] Y.-X. Liu, J. Yang, Z.-H. Zhao, C.-E. Fu and Y.-S. Duan, Phys. Rev. D 80 (2009) 065019; Y.-X. Liu, C.-E. Fu, L. Zhao and Y.-S. Duan, Phys. Rev. D 80 (2009) 065020.

[61] R. Koley, J. Mitra and S. SenGupta, Phys. Rev. D 79 (2009) 041902(R).

[62] Y. Kodama, K. Kokubu and N. Sawado, Phys. Rev. D 79, 065024 (2009).

[63] Y.-X. Liu, Z.-H. Zhao, S.-W. Wei and Y.-S. Duan, JCAP 02 (2009) 003.

[64] Y.-X. Liu, L.-D. Zhang, S.-W. Wei and Y.-S. Duan, JHEP 0808 (2008) 041.

[65] S. Aguilar and D. Singleton, Phys. Rev. D 73 (2006) 085007; M. Gogberashvili, P. Midodashvili and D. Singleton, JHEP 0708 (2007) 033.

[66] R. Jackiw and C. Rebbi, Phys. Rev. D 13 (1976) 3398.

[67] Y.-X. Liu, H.-T. Li, Z.-H. Zhao, J.-X. Li and J.-R. Ren, JHEP 0910 (2009) 091.

[68] Y.-X. Liu, H. Guo, C.-E Fu and J.-R. Ren, JHEP 1002 (2010) 080.

[69] Z.-H. Zhao, Y.-X. Liu, H.-T. Li and Y.-Q. Wang, Phys. Rev. D 82 (2010) 084030; Y.-X. Liu, C.-E. Fu, H. Guo, S.-W. Wei and Z.-H. Zhao, JCAP 1012 (2010) 031; H.-T. Li, Y.-X. Liu, 
Z.-H. Zhao and H. Guo, Phys. Rev. D 83 (2011) 045006.

[70] Z.-H. Zhao, Y.-X. Liu and H.-T. Li, Class. Quantum Grav. 27 (2010) 185001.

[71] C.-E. Fu, Y.-X. Liu and H. Guo, arXiv:1101.0336[hep-th].

[72] L. Girardello, M. Petrini, M. Porrati and A. Zaffaroni, Nucl. Phys. B569 (2000) 451.

[73] D.Z. Freedman, S.S. Gubser, K. Pilch and N.P. Warner, JHEP 0007 (2000) 038; A. Brandhuber and K. Sfetsos, Adv. Theor. Math. Phys. 3 (1999) 851.

[74] P. Breitenlohner and D.Z. Freedman, Phys. Lett. B115, (1982) 197; P. Breitenlohner and D.Z. Freedman, Ann. Phys. 144, (1982) 249. 